\title{
Interferencia gramatical en el quechua de hablantes bilingües del valle del Colca
}

\section{Grammatical interference in Quechua of bilingual speakers from Colca Valley}

\author{
Dante Callo Cuno \\ Universidad Nacional de San Agustín, Arequipa, Perú \\ Contacto: dcallo@unsa.edu.pe \\ https://orcid.org/0000-0002-1327-9878
}

\begin{abstract}
Resumen
Este artículo documenta los mecanismos que emplean los hablantes bilingües del valle del Colca en su comunicación cotidiana. A partir de evidencias, muestra unos comportamientos propios de los bilingües en el uso del quechua que se manifiesta en todos los niveles de la lengua, principalmente en el sistema gramatical. El contacto de lenguas permanente e intenso entre el español y el quechua viene provocando una serie de cambios en la estructura inmanente y trascendente de ambos idiomas. Esta conjunción de sistemas se manifiesta en una amplia gama de hechos que van desde la alternancia de códigos hasta las transferencias o interferencias, entendidas como cualquier rasgo lingüístico que se introduce en una lengua $A$ por injerencia de una lengua $B$, y debe entenderse como un fenómeno que no deteriora la estructura de las lenguas implicadas, sino más bien como una especie de enriquecimiento del sistema lingüístico.

La hipótesis de nuestra investigación determina que la interferencia gramatical en los hablantes bilingües del valle del Colca y los rasgos característicos del quechua están definidos por el contacto con el español y por las variaciones de edad, sexo y nivel de instrucción.
\end{abstract}

Palabras claves: Contacto de lenguas; Quechua arequipeño; Interferencia gramatical

\begin{abstract}
This article documents the mechanisms used by bilingual speakers from the Colca Valley in their daily communication, and shows from evidence typical behaviors of bilinguals in the use of Quechua that is manifested in all levels of the language, mainly in the grammatical system.

The permanent and intense contact of languages between Spanish and Quechua has been causing a series of changes in the immanent and transcendent structure of both languages. This conjunction of systems is manifested in a wide range of events that range from alternating codes to transfers or interferences, understood as any linguistic feature that is introduced in a language $\mathrm{A}$ by the interference of a language $\mathrm{B}$, and must
\end{abstract}


be understood as a phenomenon that does not deteriorate the structure of the languages involved, but rather as a kind of enrichment of the linguistic system. The hypothesis of our research determines that the grammatical interference in the bilingual speakers from Colca Valley and the distinguishing characteristics of Quechua are defined by the contact with Spanish and by the variations of age, sex and level of education.

Keywords: Contact of languages; Quechua from Arequipa; Grammatical interference

$$
\text { Recibido: 17.04.19 Aceptado: 23.09.19 }
$$

\section{Introducción}

El Perú es, sin duda, producto del mestizaje de las diferentes culturas que se han desarrollado en su territorio $\mathrm{y}$, sin la comprensión de este hecho, difícilmente podría explicarse su idiosincrasia. En efecto, los procesos de mestizaje que se han dado en nuestro país han sido variados y heterogéneos, diferentes en grado e intensidad y en función de factores temporales, espaciales, históricos, sociales, políticos o religiosos. La diversidad y riqueza lingüística y cultural son el efecto de este complejo proceso de enfrentamiento y fusión de culturas.

El valle del Colca, área situada en la provincia de Caylloma, Arequipa, constituye una parte de este gran espacio geográfico. Las pocas investigaciones que existen sobre el quechua arequipeño han permitido reconocer y valorar la gran diversidad cultural de la zona. Las variaciones lingüísticas que se producen en el quechua de hablantes bilingües del valle del Colca, por el contacto lingüístico entre la lengua andina e hispana, constituyen una clase de habla con una larga tradición que arranca desde la época de la conquista hasta nuestros días.

Existen numerosos trabajos sobre el español en contacto con las lenguas amerindias del país; sin embargo, tenemos pocas investigaciones sobre las influencias del español en la estructura lingüística de las lenguas nativas de la región. Por ello, aún queda mucho por hacer en este campo, y es importante entonces concentrar nuestros esfuerzos teóricos y metodológicos en el estudio del contacto de lenguas que abarquen cuestiones de descripción lingüística y explicaciones fiables y rigurosas sobre las distintas áreas del contacto de las lenguas amerindias con el español. 
La investigación se constituye en un aporte a los estudios de la lingüística del contacto, la cual en los últimos años tiene un desarrollo vertiginoso.

\section{Antecedentes, objetivos y métodos de la investigación}

Fueron hombres del clero quienes se encargaron de la documentación de las lenguas indígenas en la época colonial. Entre sus textos se encuentran fuentes indispensables para la historia de la lingüística, como el diccionario y gramática del fraile dominico Domingo de Santo Tomás (1996) y el trabajo lexicográfico del padre jesuita Diego Gonzales Holguín (1993)². Por otro lado, en el marco de la lingüística contemporánea tenemos obras históricas, descriptivas y comparativas. Nos referimos a los trabajos de dialectología quechua de Gary Parker (1963), Alfredo Torero (1964, 1974), Xavier Albo (1974), Willem Adelaar (1977), Gerald Taylor (1984) y Rodolfo Cerrón Palomino (1987).

A finales del gobierno militar de Juan Velasco Alvarado se oficializó el quechua mediante el D.L. 21156 del 27 de mayo de 1975. Al año siguiente, el Instituto de Estudios Peruanos publicó las primeras gramáticas y diccionarios sistemáticamente estructurados de los principales dialectos quechuas ${ }^{2}$. Sin embargo, no existen mayores estudios sobre la interferencia gramatical en el quechua arequipeño, pero tenemos algunas investigaciones que han sido tomadas como referencia para el desarrollo del trabajo.

Siguan (2001) ha desarrollado un capítulo sobre la interferencia lingüística, la gramática de las interferencias, actitudes ante las interferencias, etc. Es un texto de reflexión y divulgación que tiene aportes importantes en torno al tema que nos ocupa. Por otro lado, Payrató (1985) propone una concepción "neutra" con un sentido global de la interferencia al señalar que una interferencia, en sentido amplio, es un cambio lingüístico (una innovación, una pérdida, una sustitución) que se da en una lengua A (o registro), y que es motivada directamente por la influencia de una lengua B.

Así mismo, Escobar (2000) propone que el surgimiento de diversos usos lingüísticos se debe a las circunstancias socioeconómicas, políticas e históricas por las que ha atravesado nuestro país en las últimas décadas. También, Quesada 
(2007) examina aspectos referentes a la caracterización de la composición del léxico de los dialectos quechuas y propone una muestra de la reconstrucción de los elementos lexicales más tempranos a través de cambios fonéticos y semánticos.

Otro estudioso que ha realizado investigaciones importantísimas sobre lingüística del contacto es Germán de Granda (2001), quien analiza de manera acuciosa aspectos lingüísticos y sociolingüísticos del español y el quechua contemporáneos. Su taxonomía sobre la interferencia gramatical ha sido un referente importante en la estructura del presente trabajo de investigación.

Con relación al quechua arequipeño tenemos a Eva Gugenberger (1995) que estudia las actitudes lingüísticas en contextos bilingües de Arequipa; también hay algunos trabajos inéditos, entre ellos podemos citar la tesis de licenciatura de Nidia Callata y María Cervantes (1999), investigación descriptiva que ofrece una caracterización del léxico de la zona. También puede verse nuestra tesis doctoral en lingüística defendida en la UNMSM (Callo, 2015). Los objetivos de la tesis fueron identificar y explicar los rasgos gramaticales de los hablantes bilingües del valle del Colca y determinar las influencias del español en el proceso de configuración gramatical del quechua arequipeño.

La investigación es descriptiva y explicativa porque realiza un análisis sincrónico de la interferencia gramatical en el quechua de hablantes bilingües del valle del Colca y da una explicación del proceso con base en los fundamentos teóricos de la lingüística y la sociolingüística. Es descriptiva debido a que realizamos una observación sistemática de las interferencias gramaticales en el quechua del valle del Colca, indicando los casos más importantes que tendrán una valoración cualitativa. También presentamos las características de cada uno de los fenómenos de interferencia gramatical que ocurren en el quechua del valle del Colca, con el objeto de realizar el análisis correspondiente. Es explicativa porque mostramos las causas de dichas formas lingüísticas. Así mismo, damos a conocer el origen de los fenómenos de interferencia gramatical más importantes.

La población estuvo constituida por hablantes bilingües (españolquechua) del valle del Colca (Chivay, Coporaque, Yanque, Achoma, Maca y 
Cabanaconde). La muestra es representativa porque la comunidad de hablantes bilingües ha sido distribuida de acuerdo con las variables establecidas en la investigación. Sus actividades principales son la agricultura, la ganadería y el comercio; por lo general, jóvenes, padres y madres de familia mayores de edad.

Las muestras se han estructurado por cuotas de afijación uniforme. Este proceso consiste en dividir el universo relativo en subgrupos, estratos o cuotas — atendiendo a unas variables sociales determinadas - y asignar igual número de informantes a cada una de esas cuotas (tomamos como referencia la metodología del proyecto para el estudio sociolingüístico del español de España y de América, PRESEEA, del año 2003³). Las razones que nos llevaron a usar este sistema y no una muestra aleatoria o probabilística es que el trabajo por cuotas permite una comparación estadística más efectiva entre las cuotas internas de la misma y entre muestras diferentes. Además, estuvimos obligados a la búsqueda de informantes más allá de nuestros círculos que tienden a dejarse entrevistar fácilmente. Así, hemos considerado razonable crear las cuotas de la muestra a partir de tres variables sociales: el sexo, la edad y el grado de instrucción.

\section{Cuadro 1. Estructura de la muestra utilizada}

\begin{tabular}{|l|l|l|l|l|l|l|l|}
\cline { 2 - 8 } & \multicolumn{2}{l|}{$\begin{array}{l}\text { Generación 1 } \\
\text { (de 19 a 34 años) }\end{array}$} & \multicolumn{2}{l|}{$\begin{array}{l}\text { Generación 2 } \\
\text { (de 35 a 54 años) }\end{array}$} & \multicolumn{2}{l|}{$\begin{array}{l}\text { Generación } 3 \\
\text { (de 55 años a más) }\end{array}$} \\
\cline { 2 - 9 } & Hombres & Mujeres & Hombres & Mujeres & Hombres & Mujeres \\
\hline Grado de instrucción 1 & 5 & 5 & 5 & 5 & 5 & 5 \\
\hline Grado de instrucción 2 & 5 & 5 & 5 & 5 & 5 & 5 \\
\hline Grado de instrucción 3 & 5 & 5 & 5 & 5 & 5 & 5 \\
\hline
\end{tabular}

Fuente: Elaboración propia.

Referente al tamaño de la muestra, cada casilla la hemos contabilizado con una afijación uniforme de cinco informantes, por lo que el tamaño total de la muestra es de noventa informantes. Consideramos que el promedio fue razonable para el presente estudio, aunque el número de informantes fijado por PRESEEA es de tres para núcleos de menos de 500.000 habitantes; no obstante, para la investigación que realizamos optamos por seleccionar un número mayor de informantes 
con el fin de enriquecer el trabajo. Sin embargo, la población en cuestión es significativamente menor al medio millón de habitantes; todo el valle del Colca, según el último censo 2017, cuenta con una población de 86.771 personas. Al escoger 90 informantes (cinco por cuota) obtenemos una representatividad de 1/2234, que supone el $0,07 \%$ de la población, bastante superior al nivel canónico $(0,025 \%$ de representatividad propuesto por Labov).

Para que un informante pudiera ser considerado válido debía cumplir con un requisito más: haber nacido en el valle del Colca o tener una residencia mayor a quince años.

\section{Lenguas en contacto}

Las lenguas en contacto están definidas como el discurso de la comunicación entre dos comunidades lingüísticas. Según Weinreich "dos lenguas están en contacto si se usan alternadamente por las mismas personas" (1953, p. 1), por ejemplo, el caso de los hablantes bilingües (español-quechua) del valle del Colca.

Para estudiar las lenguas en contacto se deben considerar dos tipos de factores: los intrínsecos, que se refieren a la estructura y la relación entre las lenguas; y los extralingüísticos, que analizan las condiciones sociales. Por ello, para examinar el fenómeno de la interferencia (en un ambiente bilingüe), no son suficientes los estudios puramente lingüísticos de las lenguas, pues se deben incluir otros factores, los extralingüísticos.

El contacto de lenguas genera una serie de variedades lingüísticas. Estas variedades constituyen un conjunto de modalidades expresivas que se usan en diversas comunidades de habla; llegan a adquirir en muchas ocasiones el valor de variantes diastráticas o diafásicas y forman parte de un sistema pragmalingüístico condicionado.

La situación lingüística en nuestro país, tomando en cuenta toda su problemática, es una situación de bilingüismo social diglósico. En la región Arequipa se usan dos lenguas en la comunicación convencional: el quechua y el español; la primera es minoritaria y usada de manera informal; mientras que 
el español es el idioma oficial y mayoritario, el único a ser usado en situaciones formales.

El término interferencia admitiría dos usos o interpretaciones diferentes. En un sentido amplio, designaría el proceso general de la influencia que las lenguas ejercen entre sí como consecuencia de las situaciones de contacto. Y en un sentido más restringido, podría emplearse también para designar las diferentes fases por las que atraviesa dicha influencia.

Los sistemas gramaticales de cada lengua son distintos, y las diferencias entre ellos son a su vez distintas en cada par de lenguas, por lo que las interferencias serán, en consecuencia, distintas.

Realmente es muy difícil hablar de tipos de interferencia gramatical; sin embargo, con base en la taxonomía que hace el lingüista Granda (2001), distinguimos en la interferencia gramatical dos grandes campos:

a) La interferencia gramatical cuantitativa se caracteriza por la modificación del inventario de rasgos gramaticales existentes en la lengua objetivo, tanto por adopción de uno nuevo, procedente de la lengua fuente, como por eliminación de uno propio:

$\begin{array}{ll}\text { awichuy } & \text { 'abuelo' } \\ \text { awichay } & \text { 'abuela' }\end{array}$

b) La interferencia gramatical cualitativa actúa en la lengua objetivo por sustitución de elementos estructurales de aquella por otros, de función idéntica, procedentes de la lengua fuente y en otros por reestructuración de rasgos de una lengua objetivo:

warmi 'mujer' warmi-s 'mujeres'
chuwa chuwa-s 'platos'
nuqaykuta manan qhawarinwankupashchu
'a nosotros no nos miran siquiera'




\section{Aspectos geográficos y sociolingüísticos del valle del Colca}

El quechua arequipeño que examinamos es hablado en la provincia de Caylloma, ubicada en el extremo nororiental de la región Arequipa en el sur del país. Tiene una extensión total de $14.780 \mathrm{~km}^{2}$ distribuidos en 20 distritos, lo que equivale al $27,07 \%$ de la superficie total. Su población actual, según el censo del año 2017, es de 86.771 habitantes.

Partiendo de la ciudad de Arequipa (a 2363 msnm en la zona quechua), a $160 \mathrm{~km}$ se encuentra el valle del Colca, un lugar hermoso y apacible. Accesible por un camino que bordea las faldas del Chachani, pasando por zona de punas, la meseta de Vincocaya, la pampa de Toccra y estrechos desfiladeros, se arriba a los altos desde donde se divisa el pueblo de Sibayo. El río que nace a una altura de 5000 metros en la zona de Condoroma y desemboca en el océano Pacífico se le conoce con el nombre de río Camaná.

El valle del Colca es el corazón de la provincia de Chivay, que a la vez es el centro del quechua arequipeño. Es un valle de riqueza agronómica, arquitectónica y tradicional, en el que el hombre vive en relación íntima con la tierra.

En estas punas del Perú profundo existen abundantes pastos naturales propicios para la crianza de ganado. Toda esta región se levanta sobre los 3000 metros de altitud. El río principal en esta región es el Colca, que nace en Crucero Alto, y el Tarucani que se desliza por lo más profundo de la quebrada del mismo nombre (Pease, 1977, pp. 152, 157, 170).

\subsection{Collaguas y Cabanas}

En 1586, Juan Ulloa Mogollón ${ }^{4}$ había observado que los habitantes de Cabanaconde eran diferentes a los demás collaguas de las partes altas (1965, p. 327). No solo por la mayor fertilidad de sus tierras, sino por razones esencialmente lingüísticas. Mientras los pobladores de las zonas más bajas y cálidas de Cabanaconde hablaban quechua, los hombres de las tierras más altas y frías de Yanque, Lari, Tuti, etc., hablaban aimara.

Según Manrique es evidente la existencia de dos etnias diferenciadas que 
ocupan el valle: los collaguas en la zona alta del curso del Colca y los cabanas en la zona baja (1985, p. 31). Las diferencias entre ambos grupos eran claramente perceptibles aun a fines del siglo XIV, como se desprende de la visita realizada en 1586 por Ulloa Mogollón. Estas se manifestaban, en primer lugar, en la zona que ambas etnias reconocían como pacarina (o pacarisca: lugar de origen).

Los collaguas decían proceder del volcán situado en Vellille, denominado Collaguata. De este derivaba su nombre. Los cabanas, en cambio, reconocían como pacarina el nevado Wallka allka, de donde, derretida la nieve, se aprovechan del agua para sus tierras de regadío. Los cabanas habían asumido la denominación de Cabanaconde (Kunti).

Ambas etnias reconocían haberse instalado en el valle del Colca luego de expulsar por las armas a sus antiguos pobladores. Collaguas y cabanas eran diferentes, además, en vestimenta, lenguaje e incluso en su aspecto físico; esto último debido al uso de diversas deformaciones craneanas. Los collaguas apretaban la cabeza a los niños tan reciamente que se la ahusaban y adelgazaban, de manera alta y prolongada, lo más que podían, pues las cabezas debían tener la forma alta del volcán de donde salieron. Los cabanas, en cambio, ataban la cabeza a los recién nacidos para hacerla ancha y desproporcionada.

Como dijimos anteriormente, es importante indicar las diferencias lingüísticas de ambas etnias. Los collaguas hablaban aimara y los cabanas el quechua cusqueño; aunque existían también otros idiomas locales: el puquina, el coli y el isapi. Torero sugiere que estas últimas lenguas debían ser variedades del puquina, la tercera lengua general en importancia en el período (1972, p. 74). La presencia del aimara en la zona no nos debe extrañar, puesto que esta lengua se hablaba aun en Vilcashuamán (Ayacucho). Así, su presencia no implica una relación con el Collao, como suponen algunos autores. Torero ha demostrado que el aimara se originó en el eje geográfico Nazca-Ayacucho de un tronco lingüístico originario denominado por el lingüista huachano Aru. De este derivaron el aimara (que avanzó hacia el sur y penetró el Collao, obligando a replegarse al puquina recién hacia el siglo XII d. C.) y dos idiomas que existían, aunque en proceso de extinción, en la provincia de Yauyos: el cauqui y el jaqaru. 
Actualmente pocos hablan la lengua aimara en esta zona, pero los nombres de una gran parte de las comunidades y poblados del valle del Colca tienen dicho origen. Mas la uniformación lingüística en el valle del Colca en torno al quechua es un fenómeno mucho más reciente de lo que generalmente se cree.

\subsection{El quechua del valle del Colca}

Torero clasifica al quechua de Arequipa junto con el quechua "cusqueñoboliviano", aunque sostiene que no todo se ha descubierto. La divergencia entre sus variedades del cusqueño-boliviano es probablemente mayor de lo que aparece en los estudios comparativos hasta el momento (Torero, 1974, p. 35). Además, esta variedad quechua se habla en la mitad de la región Apurímac hacia el sur; la región Cusco; las provincias altas de Arequipa: La Unión, Condesuyos, Castilla y Caylloma; las provincias de Carabaya, Melgar, Azángaro, la parte occidental de Sandia, Puno y Lampa, en la región Puno; la provincia de Sánchez Cerro en Moquegua y gran parte del territorio boliviano, cuyas irrupciones modernas llegaron a Salta y Jujuy en Argentina y Antofagasta en Chile.

Cusihuamán reconoce la variación notoria en el quechua que él denomina Cusco-Collao pero, como otros, no le da mayor importancia (1976, p. 32). Declara que dicha variedad abarca las regiones de Cusco, Arequipa, Puno y unas partes de Apurímac, y que el sistema fonológico y la estructura gramatical de este quechua son homogéneos.

El quechua del valle del Colca es una variedad dialectal parecida en ciertos aspectos al quechua cusqueño y, en otros, al quechua ayacuchano. Sus diferencias son más morfofonológicas que gramaticales. Veamos: las terminaciones -chis (Cusco) y -chik (Caylloma); el uso del grafema / $h /$ en reemplazo del fonema semivocal /y/: haykuy, yaykuy; presencia de fonemas adicionales: allpa, hallpa; la utilización del morfema del pretérito imperfecto -ra: maskharanku mikhunata, 'buscaban comida'; maskharanku qurpachanata, 'buscaban alojamiento'.

El quechua del valle del Colca tiene mucha influencia del aimara porque la mayor parte de los topónimos de la zona tiene este origen. Ello se puede explicar porque a inicios del siglo XVII, los collaguas que habitaban los pueblos de Maca 
hasta Tisco hablaban aimara. Así mismo, igual que en el quechua cusqueño los hablantes del valle del Colca usan los fonemas oclusivos, africados y glotalizados ( $p^{\prime}, t^{\prime}, k^{\prime}, q^{\prime}, p h$, th, $k h, q h, c h, c h$ ', chh): sumaq rit'i umayuq 'majestuosa nieve en la cabeza', hukniraq sach 'aman tukun 'a veces se convierte en árbol'.

En tal sentido, clasificaremos las variedades del quechua arequipeño con base en criterios etnológicos y geográficos.

\subsection{Las variedades arequipeñas}

Las dos concentraciones mayores de quechuahablantes, fuera de la ciudad de Arequipa, se encuentran en los valles de los ríos Colca y Cotahuasi. El quechua se habla también en las comunidades esparcidas en el Altiplano, donde es el idioma predominante en el norte del departamento. Las tres variedades que se hablan en Arequipa corresponden a las regiones mencionadas: el quechua arequipeño de altura (QAA), el quechua arequipeño central (QAC) y el quechua arequipeño occidental (QAO).

El valle del Colca, en el centro de la provincia de Caylloma, presenta una muestra de las tres variedades del quechua arequipeño. El valle se divide naturalmente en tres zonas ecológicas mayores que también marcan las divisiones dialectales: la zona alta, con la variedad QAA; la zona central, con la variedad QAC; y la zona baja, donde se habla la variedad QAO.

La variedad QAA es usada mayoritariamente por toda la comunidad de hablantes, aquí mucho tiene que ver la ubicación geográfica de los poblados del valle del Colca. En los distritos de Callalli, Sibayo, Tisco y Caylloma, el uso de la lengua se mantiene en todos los ámbitos. Hemos observado que en la plaza de Callalli los pocos comerciantes que venden chicha y algunos otros alimentos emplean el quechua como medio para el intercambio de mensajes. También en el campo, en la chacra y en la casa solo usan quechua, tanto mayores como jóvenes. En el trabajo de campo, al visitar a una de las familias en Callalli, hemos observado y escuchado el diálogo de Melchor (62 años) y Antonia, su esposa (58 años). Veamos:

MelCHOR : Runakuna hamusqachu manachu

'Los hombres vinieron o no' 


\begin{tabular}{|c|c|}
\hline ANTONIA & $\begin{array}{l}\text { Ari, hamurqanku ratulla } \\
\text { 'Sí, han venido rápido' }\end{array}$ \\
\hline MELCHOR & $\begin{array}{l}\text { Pitaq, kay q'ariri } \\
\text { 'Quién es este hombre' }\end{array}$ \\
\hline ANTONIA & $\begin{array}{l}\text { Manan riqsinichu, ususinchis aparqamuska } \\
\text { 'Yo no lo conozco, creo que es amigo de nuestra hija' }\end{array}$ \\
\hline MELCHOR & $\begin{array}{l}\text { Imata munan } \\
\text { 'Qué quiere' }\end{array}$ \\
\hline ANTONIA & $\begin{array}{l}\text { Manan yachanichu } \\
\text { 'No sé' }\end{array}$ \\
\hline
\end{tabular}

\section{Callalli, 15.06.2010}

Como podemos observar, el diálogo entre los participantes ha sido en quechua. No encontramos ningún tipo de préstamos; mantiene su pureza gramatical, fonética y léxica. Además, su uso mayoritario entre los pobladores de las zonas altas, se debe a las exigencias que hacen los mayores de mantener el quechua en la comunicación con las nuevas generaciones.

Los hablantes de Chivay reconocen que en las partes altas —dígase: Caylloma Callalli, Sibayo, etc.-, los comuneros solo hablan quechua. Así lo hemos constatado en nuestras visitas; en todo caso, veamos lo que dice un hablante de Chivay:

Punapin allinta rimakunku runisimita; kaypiqa masta castillanullata puniya rimakunku.

'En la puna hablan bien el quechua. Aquí se habla más el castellano'.

\section{Chivay, 20.11.2009}

Como hemos podido observar, los señores que hablan usan indistintamente el quechua y el español. Cuando no encuentran la palabra quechua en su real dimensión —a veces desconocen su existencia—, usan el español rápidamente. Se trata de hablantes bilingües.

El uso de la variedad QAA tiene un elevado índice de identificación y lealtad a la lengua de sus mayores o ancestros. 
Los hablantes de la variedad QAC usan de manera alternada el quechua y el español. Observamos el diálogo de dos personas en la iglesia de Chivay, cuando conversaban sobre el inca Maita Cápac:

kay llaqtamanmi inka Mayta Qhapaq Chayamusqa. Kaypi karqan llampu runakunalla, pisilla tiyakurqanku.

'Cuando el Inca Maita Cápac llegó a este pueblo con todos sus guerreros, aquí vivían pocos hombres y eran pacíficos'.

\section{Chivay, 10.06.2010}

Es importante indicar que los diferentes grados de predomino de uso del quechua en el valle del Colca se deben a la ubicación geográfica, la edad de los hablantes y la lealtad lingüística. En tal sentido, la historia etnológica y lingüística del Colca ayuda a comprender la razón de las diferencias que existen hasta hoy en las variedades del Colca.

\section{a) La variedad $Q A A$}

Es la variedad hablada en las zonas altas de Arequipa, al norte y en el extremo oriental de este departamento. Abarca toda la parte alta de la región y se extiende desde los límites con Apurímac y Ayacucho por el noroeste (incluyendo Puica), hasta Chachas y Choco en la orilla oriental del río Orcopampa, las cabeceras del río Colca, y Tarucani, Pati y los demás pueblos en los límites con Moquegua.

En el Colca, el QAA tiene presencia en los pueblos y las comunidades desde Callalli, Pulpera y Tisco hacia arriba. Las actividades y las costumbres de los habitantes de esta zona tienen su origen en las condiciones climatológicas y de altitud que no les permiten el cultivo de la tierra. Son pastores, artesanos y comerciantes: migrantes que pastan animales en lugares alejados del pueblo la mayor parte del año.

Los vocablos que usan los comuneros de esta zona se ven menos interferidos por el español. El quechua mantiene su pureza lingüística en el aspecto fonético y fonológico, morfosintáctico y léxico. 


\section{b) La variedad $Q A C$}

Tradicionalmente, la región donde se habla el QAC, con la mayor concentración de población quechua del valle, ha sido el centro de influencia en la parte nororiental de región Arequipa.

En esta zona la mayor parte de los habitantes se dedica a la agricultura y la ganadería, en algunos casos al comercio y al turismo. Viven en centros poblados importantes, entre ellos los distritos de Sibayo, Tuti, Chivay, Yanque, Coporaque, Achoma, Ichupampa, Lari, Maca, Cabanaconde y Madrigal. Coporaque es reconocido como el primer centro poblado del Colca; Yanque se estableció como el centro político Collao-Incaico y la primera capital provincial; y Chivay es el actual centro político y comercial de la provincia de Caylloma.

\section{c) La variedad $Q A O$}

La variedad arequipeña que tiene más rasgos comunes con el dialecto ayacuchano, lógicamente se encuentra en la frontera occidental de Arequipa. Empezando en la provincia de La Unión, se extiende hacia el sur en forma de una herradura siguiendo los valles de las provincias de Condesuyos y Castilla.

Desde Alca baja por Cotahuasi, abarca Sayla, Salamanca-Chichas, Yanaquihua y Chuquibamba. La frontera nororiental empieza en Orcopampa y comprende los distritos ubicados en la margen occidental del río Orcopampa hasta los pueblos cercanos a Cabanaconde, Pinchollo, Yanque y Tapay, en el valle del Colca.

\subsection{Fonología del quechua del valle del Colca: fonemas consonánticos y vocálicos}

El sistema fonológico del QAC consta de treinta y un fonemas segmentales que se agrupan de la siguiente manera: cuatro oclusivas sordas simples, $/ p, t, k, q /$ que hacen contraste con dos series paralelas de oclusivas: las oclusivas sordas glotalizadas / $p^{\prime}, t^{\prime}, k^{\prime}, q^{\prime} /$ y las oclusivas sordas aspiradas $/ p h, t h, k h, q h /$; la africada sorda simple $/ \mathrm{ch} /$ que hace contraste con la africada glotalizada $/ \mathrm{ch}$ ' y la africada aspirada $/ \mathrm{ch} h /$; tres fricativas sordas $/ s, s h, h /$; tres nasales sonoras $/ m, n$, $\tilde{n} /$; dos laterales $/ l, l l /$; una vibrante $/ r /$; dos semiconsonantes sonoras $/ w, y /$; dos vocales altas, $/ i$, $u /$; y una baja $/ a /$ : 


\begin{tabular}{|c|c|c|c|}
\hline chaqui & 'pie' & churay & 'poner' \\
\hline kaniy & 'morder' & takiy & 'cantar' \\
\hline china & 'hembra' & kamay & 'ordenar' \\
\hline pakay & 'esconder' & killa & 'luna' \\
\hline tuta & 'noche' & uma & 'cabeza' \\
\hline chhaqay & 'aquel o aquella' & phaway & 'correr' \\
\hline khituy & 'raspar' & thanta & 'trapo' \\
\hline chhuqay & 'arrojar' & khamuy & 'masticar' \\
\hline qhatu & ‘mercado' & thuqay & 'escupir' \\
\hline phukuy & 'soplar' & ch'aki & ‘seco’ \\
\hline qhuña & 'moco' & ch’uño & ‘chuño’ \\
\hline k'amiy & 'reñir' & q'asa & 'desportillado' \\
\hline pákiy & 'romper' & q'uillu & 'amarillo' \\
\hline$t^{\prime} i k a$ & 'flor' & punku & 'puerta' \\
\hline t'anta & 'pan' & qankuna & 'ustedes' \\
\hline
\end{tabular}

El cuadro 2 muestra los fonemas consonánticos del QAC con sus respectivos puntos de articulación:

Cuadro 2. Fonemas consonánticos del quechua arequipeño

\begin{tabular}{|l|c|c|c|c|c|c|}
\hline \multicolumn{1}{|c|}{ CONSONANTES } & Labial & Alveolar & Palatal & Velar & Posvelar & Glotal \\
\hline Oclusivas simples & $p$ & $t$ & & $k$ & $q$ & \\
\hline Oclusivas glotalizadas & $p^{\prime}$ & $t^{\prime}$ & & $k^{\prime}$ & $q^{\prime}$ & \\
\hline Oclusivas aspiradas & $p h$ & $t h$ & & $k h$ & $q h$ & \\
\hline Africadas simples & & & $c h$ & & & \\
\hline Africadas glotalizadas & & & $c h '$ & & & \\
\hline Africadas aspiradas & & & $c h h$ & & & \\
\hline Fricativas & & $s$ & $s h$ & & & $h$ \\
\hline Nasales & & $l$ & $l l$ & & & \\
\hline Laterales & & $r$ & & & & \\
\hline Vibrante & $w$ & & $y$ & & & \\
\hline Semivocales & & & & & & \\
\hline
\end{tabular}

Fuente: Elaboración propia. 
Cuadro 3. Fonemas vocálicos del quechua arequipeño

\begin{tabular}{|l|c|c|c|}
\hline VOCALES & Anterior & Central & Posterior \\
\hline Altas & $i$ & & $u$ \\
\hline Medias & & & \\
\hline Bajas & & $a$ & \\
\hline
\end{tabular}

Fuente: Elaboración propia.

\section{La interferencia gramatical en el quechua del valle del Colca}

El ámbito de la gramática ha sido el menos estudiado desde el punto de vista de la interferencia, déficit que resulta fundamental en la exploración de los fenómenos en los que el quechua convive con el español. El contacto de las dos lenguas en esta comunidad de habla permite que los lugareños conjuguen una serie de recursos lingüísticos que sirven para optimizar la comunicación. Por ello identificamos la estructura y los tipos de interferencia gramatical cuantitativa y cualitativa para el análisis correspondiente.

\subsection{Contacto quechua-español en el valle del Colca}

El quechua de esta zona se caracteriza sociolingüísticamente por un intenso fenómeno diglósico con el español como modalidad $\mathrm{H}$ y por un notorio proceso de retracción, tanto de extensión como de uso, por el cual la forma española de comunicación se configura no solo socialmente como lengua hegemónica, sino también, a escala individual, como variedad de predominio; de tal forma, sus estructuras gramaticales funcionan incluso a manera de contextos referenciales en el quechua local.

El contacto de lenguas en el valle del Colca entre el español y el quechua hace que el bilingüismo sea intenso, lo que ha dado origen a una serie de interferencias o transferencias lingüísticas de la lengua fuente a la lengua objetivo. Estas marcas transcódigas deben entenderse como cualquier rasgo lingüístico que se introduce en una lengua A por la influencia de una lengua B, como observamos en el análisis de las secuencias lingüísticas idiosincrásicas que configuran la variedad del quechua del valle del Colca. Así, el hablante emplea piezas o construcciones que son diferentes a los usos del quechua patrimonial. 
La producción lingüística de un bilingüe no puede ser evaluada como si fuera la de un monolingüe; el bilingüismo no es la suma de dos monolingües, sino que tiene una particular configuración lingüística a partir de la que ha desarrollado la competencia comunicativa, suficiente para sus interacciones diarias, marcada por la coexistencia de las lenguas.

Referente al contexto lingüístico familiar y social, indicamos que el ámbito como conjunto de factores que hacen posible la comunicación tiene que ver con la organización social como base conceptual. Fishman (1965) toma como punto de partida las preguntas ¿quién habla, qué lengua, con quién y cuándo? Con este enfoque, los hablantes bilingües del valle del Colca en la casa hablan 93\% quechua y solo usan de manera ocasional el español (7\%). En el pueblo, con sus amigos, $63 \%$ de hablantes emplea el quechua y el 37\% utiliza el español. En la chacra, las cifras se elevan, porque el $97 \%$ habla quechua y el 3\% lo hace en español. Ello debido a que la chacra es un espacio privilegiado para las manifestaciones comunales, el cual se relaciona con la productividad o el sustento de vida. No debemos olvidar que en la época de siembra y cosecha es el espacio que congrega a gran cantidad de gente.

Aquí observamos niveles de interacción comunicativa como el contexto y el evento social. Los hablantes bilingües del valle del Colca, en la casa y en la chacra, se interrelacionan de manera notable en quechua porque reconocen el escenario como suyo y los interlocutores se identifican plenamente con el evento social, por lo que resuelven interactuar en la lengua de sus mayores. No obstante, usan indistintamente las dos lenguas en toda situación, ámbito o acto comunicativo. La situación comunicativa tiene que ver con el contexto o el escenario social dentro del cual ocurre la comunicación, el evento constituye la unidad básica para propósitos descriptivos, ya que se refiere a toda actividad que está gobernada por reglas o normas de uso del habla.

En tal sentido, podemos considerar que el uso de la lengua española aumenta en el pueblo porque las personas acuden con mayor frecuencia a las instituciones, sea por asuntos legales, comerciales, educativos, de salud u 
otros. Así, el 94\% de informantes bilingües en el valle del Colca utiliza la lengua española; esto se debe indudablemente al contacto de lenguas entre estas comunidades de hablas diferentes. Aquí surgen una serie de fenómenos lingüísticos y extralingüísticos: interferencia, alternancia, etc., es decir, los hablantes bilingües emplean elementos del español cuando se interrelacionan en quechua. Estos signos pueden ser palabras, sintagmas o unidades mayores. Usan palabras del español en el quechua porque desconocen el término o concepto específico para un determinado tema; en otros casos, estos usos se dan también cuando el hablante bilingüe alterna un código con otro, mostrando así sus habilidades lingüísticas.

Finalmente, cuando se les preguntó a los hablantes bilingües del valle del Colca sobre cuál lengua preferían para la enseñanza de sus hijos, las respuestas corroboraron que el quechua mantiene su prestigio y vitalidad lingüística: 92\% de hablantes considera bueno que la educación de sus hijos se dé en ambas lenguas, hecho que resulta importante pero que constituye un reto para las autoridades del Ministerio de Educación o la Gerencia Regional de Educación de Arequipa.

Siguiendo las pautas clasificatorias establecidas, en una serie de trabajos de investigación desarrollados por Granda (2001), analizamos los fenómenos de interferencia en el quechua del valle del Colca. Thomason y Kaufman (1988) demostraron que no existen restricciones tipológicas ni gramaticales para los fenómenos de interferencia lingüística. Por ello, y con base en la información empírica en nuestro poder, estamos de acuerdo con las principales conclusiones que señala Granda (2001):

a) No existe ningún tipo de restricción interna a los fenómenos de interferencia que derivan del contacto de lenguas.

b) La extensión y profundidad de los mismos están condicionadas exclusivamente por factores de índole sociolingüística relacionados, primordialmente, con la duración, intensidad y modalidades de la situación de coexistencia o de la relación que mantienen entre sí las comunidades humanas que manejan las lenguas en cuestión.

c) No es admisible, metodológicamente, la anteposición sistemática de los factores internos o los externos para la determinación de los 
elementos condicionadores de los fenómenos de cambio lingüístico. Una explicación externa derivada en muchas ocasiones del proceso de contacto lingüístico fuerte y unificada es preferible a otras, de índole interna, débil o múltiple (p. 300).

Los fenómenos de interferencia gramatical pueden ser de índole cuantitativa, que conllevan la modificación del inventario de rasgos gramaticales existentes en la lengua objetivo (bien por adopción de uno nuevo, procedente de la lengua fuente, bien por eliminación de uno propio); y los de carácter cualitativo, que no varían cuantitativamente dicho inventario, aunque sí alteran alguno o algunos de sus elementos constitutivos (Granda, 2001, p. 304). Estas formas transcódigas pueden ser unidireccionales o bidireccionales, ya que las influencias van y vienen entre las lenguas correspondientes.

Como observaremos, el quechua del valle del Colca presenta una serie de casos de interferencia gramatical que examinaremos a continuación.

\subsection{Interferencia gramatical cuantitativa}

La interferencia gramatical cuantitativa se caracteriza por la aparición de morfemas en la lengua objetivo (quechua) por influencias del español contemporáneo o de la lengua fuente. Estas formas pueden ser por adopción de uno nuevo, procedente de la lengua fuente o por eliminación de uno propio.

\section{a) Interferencia por adopción}

Este fenómeno de interferencia gramatical cuantitativa ocurre cuando un rasgo estructural de la lengua fuente incorpora a la lengua objetivo marcas morfológicas de género presentes en la lengua rectora o dominante, pero inexistentes en la lengua receptora o subordinada.

El quechua patrimonial no cuenta con morfemas de género, es decir, las palabras no tienen flexión para el masculino y el femenino como en el español (gato, gata; alumno, alumna). El sexo de los seres se distingue mediante los modificadores o elementos adyacentes warmi, 'mujer' y qhari, 'varón'; warmiwawa, 'niña' y qhariwawa, 'niño' cuando se trata de personas. Cuando se denotan animales se usan 
los modificadores china, 'hembra' y urqu, 'macho' (china allqu, 'perra'; urqu allqu, 'perro'). Se emplean también palabras especiales que se refieren específicamente, ya sea a seres de sexo femenino o masculino: paya, 'vieja'; machu, 'viejo'; ñaña o pana, 'hermana'; turi o wawqi, 'hermano' (Soto, 2006).

El género es una propiedad de los nombres que sirve para diferenciar el sexo del referente. Como ya dijimos en el párrafo anterior, el quechua no tiene marca para indicar el morfema analítico de género como se hace en el español, pero gramaticalmente aporta información semántica en los nombres que designan seres animados, ya que diferencia el sexo que les corresponde.

En el quechua del valle del Colca se vienen utilizando los morfemas de género -o / -a (-u / -a), para marcar la oposición masculino/femenino. Se añade al morfema lexemático quechua los morfemas flexivos que corresponden a la lengua fuente. Estas formas son 뜨 para el masculino y a para el femenino:

$\begin{array}{ll}\text { (1) awichuy hamusqa } & \text { 'mi abuelo ha venido' } \\ \text { awichay waqan } & \text { 'mi abuela llora' } \\ \text { niñucha purin llaqtata } & \text { 'el niño va al pueblo' } \\ \text { niñacha kutimusqa } & \text { 'la niña ha regresado' } \\ \text { mayistru macharqan } & \text { 'el maestro había marchado' } \\ \text { mayistra purisqa Makaman } & \text { 'la maestra había ido a Maca' } \\ \text { huwis purin plasata } & \text { 'el juez va a la plaza' } \\ \text { huwisa munay puni kasqa } & \text { 'la jueza es muy bonita' }\end{array}$

Como podrá observarse, la mayor parte de los sustantivos que acaban en - $\boldsymbol{a}$ son femeninos (awichay, niñacha, mayistra, etc.) y la mayoría de los que lo hacen en $\boldsymbol{-} \boldsymbol{u}$ son masculinos (awichuy, niñucha, mayistru, etc.) Al igual que en la lengua española, los que terminan en consonante pueden ser masculinos o femeninos (huwis y huwisa).

Por otro lado, como se observa en las oraciones (2), el género masculino 
y femenino no solo aparece en nombres comunes, sino también en la estructura de nombres propios:

(2) Alwirtu mikhun sarapilata

Alwirta puñushan

Antuku hamunqa

Antuka chayamun
'Alberto come mazamorra de maíz'

'Alberta duerme'

'Antonio vendrá'

'Antonia ha llegado'

Al igual que en la lengua española, en el quechua del valle del Colca algunos nombres adoptan terminaciones específicas para el femenino:

(3) misti purinqa Limata mistisa takisunki 'el joven irá a Lima'

'la joven te cantará'

El nombre común animado misti, está en género masculino y se refiere a una persona que no es del lugar y que aparentemente es de una condición económica importante; mistisa, es un nombre común femenino. Estas dos palabras se usan frecuentemente en el habla del valle del Colca. El fomento y desarrollo del turismo en el valle del Colca ha hecho que los hablantes usen algunos neologismos en su interrelación cotidiana:

(4) huk rinku takiran munayta tawa rinkakuna takirqanku 'un gringo cantaba bonito'

'cuatro gringas han cantado'

Los nombres comunes rinku, rinka (gringo/-a en lengua española), como es natural, expresan el género masculino y femenino, respectivamente. El contacto de lenguas en el valle del Colca hace que con frecuencia se incremente el vocabulario del quechua de la región, formando nuevas estructuras gramaticales que enriquecen la competencia lingüística del hablante bilingüe. Es decir, producto de la influencia de la lengua dominante en esta comunidad de habla, los bilingües usan los mismos procedimientos que se tienen en lengua española para formar el género correspondiente. 


\section{Cuadro 4. Incidencia de interferencia gramatical cuantitativa por adopción}

\begin{tabular}{|c|c|c|c|c|}
\hline \multicolumn{5}{|c|}{$-\mathbf{u},-\mathbf{a} / \mathbf{- \theta}$} \\
\hline & \multicolumn{4}{|c|}{$\begin{array}{c}\text { Generación } 1 \\
\text { (de } 19 \text { a } 34 \text { años) }\end{array}$} \\
\hline & \multicolumn{2}{|c|}{ Hombre } & \multicolumn{2}{|c|}{ Mujer } \\
\hline & $-\mathrm{u},-\mathrm{a}$ & $-\theta$ & $-\mathrm{u},-\mathrm{a}$ & $-\theta$ \\
\hline Primaria & $90 \%(18)$ & $10 \%(2)$ & $80 \%(16)$ & $20 \%(4)$ \\
\hline Secundaria & $90 \%(18)$ & $10 \%(2)$ & $90 \%(18)$ & $10 \%(2)$ \\
\hline Superior & $80 \%(16)$ & $20 \%(4)$ & $80 \%(16)$ & $20 \%(4)$ \\
\hline
\end{tabular}

Fuente: Elaboración propia.

El cuadro 4 permite observar que el uso de morfemas de género en hablantes bilingües del valle del Colca tiene una incidencia importante debido a que el español influye notablemente en la interrelación humana.

Observamos que en la generación 1 (19 a 34 años), la incidencia de uso de los morfemas de género españoles -o, -a / -u, -a es elevadísima tanto en hombres como en mujeres. En el primer caso alcanza 90\% y, en el segundo, 80\%. Aquí se nota que, pese a la igualdad de condiciones sociales y situacionales en esta comunidad lingüística, el habla de las mujeres es diferente al habla de los hombres. En la variable que indica el nivel de instrucción - primaria, secundaria y superior-, también el uso del morfema español es alto, entre $80 \%$ y $90 \%$ respectivamente.

Este procedimiento, que es común en el lenguaje de los hablantes bilingües del valle del Colca, no es visto por los usuarios como algo negativo. 


\section{Cuadro 5. Incidencia de interferencia gramatical cuantitativa por adopción} $-\mathbf{u},-\mathbf{a} /-\boldsymbol{\theta}$

\begin{tabular}{|c|c|c|c|c|}
\hline & \multicolumn{4}{|c|}{$\begin{array}{c}\text { Generación } 2 \\
\text { (de } 35 \text { a } 54 \text { años) }\end{array}$} \\
\hline & \multicolumn{2}{|c|}{ Hombre } & \multicolumn{2}{|c|}{ Mujer } \\
\hline & $-\mathrm{u},-\mathrm{a}$ & $-\theta$ & $-\mathrm{u},-\mathrm{a}$ & $-\theta$ \\
\hline Primaria & $90 \%(18)$ & $10 \%(2)$ & $90 \%(18)$ & $10 \%(2)$ \\
\hline Secundaria & $90 \%(18)$ & $10 \%(2)$ & $80 \%(16)$ & $20 \%(4)$ \\
\hline Superior & $80 \%(16)$ & $20 \%(4)$ & $80 \%(16)$ & $20 \%(4)$ \\
\hline
\end{tabular}

Fuente: Elaboración propia.

Se observa que el uso del morfema de género español -o, -a / - u, -a se impone también en la generación 2 (35 a 54 años). Así, el 90\% corresponde a hombres y mujeres de nivel primario. En el nivel secundario la incidencia es de $90 \%$ en los hombres y $80 \%$ en las mujeres. En esta generación, los hombres vienen adoptando los rasgos morfológicos del español con mayor intensidad; las mujeres, en cambio, utilizan estas formas en menor proporción. Ello demuestra que el comportamiento lingüístico de hombres y mujeres en la conversación o en la interrelación humana es diferente, así se observa en esta comunidad de habla; es decir, el análisis sociolingüístico que realizamos en el valle del Colca nos indica que el sexo es uno de los factores extralingüísticos preeminentes en la interferencia gramatical. En el nivel superior, 80\% de hombres y mujeres adoptan el morfema genérico de la lengua española.

Cuadro 6. Incidencia de interferencia gramatical cuantitativa por adopción

\begin{tabular}{|l|c|c|c|c|}
\cline { 2 - 5 } \multicolumn{1}{c|}{} & \multicolumn{4}{|c|}{$-\mathbf{u},-\mathbf{- a}-\mathbf{- \theta}$} \\
\cline { 2 - 5 } & \multicolumn{3}{|c|}{$\begin{array}{c}\text { Generación 3 } \\
\text { (de 55 años a más) }\end{array}$} \\
\cline { 2 - 5 } \multicolumn{1}{c|}{ Hombre } & $-\theta$ & $-\mathrm{c},-\mathrm{a}$ & $-\theta$ \\
\hline Primaria & $-\mathrm{u},-\mathrm{a}$ & $100 \%(20)$ & $0 \%(0)$ & $100 \%(20)$ \\
\hline Secundaria & $0 \%(0)$ & $90 \%(18)$ & $0 \%(0)$ & $100 \%(20)$ \\
\hline Superior & $10 \%(2)$ & $100 \%(20)$ & $0 \%(0)$ & $100 \%(20)$ \\
\hline
\end{tabular}

Fuente: Elaboración propia. 
Finalmente, los datos de estos cuadros confirman efectivamente la mayor frecuencia de los morfemas genéricos $-u$, para el masculino y - $a$ para el femenino. Estas marcas de género de la lengua española alcanzan su mayor dimensión en hombres y mujeres de la primera y segunda generación; en el primer caso, se observa que $90 \%$ de hablantes bilingües del valle del Colca (hombres) utiliza el morfema genérico español $-u,-a$; y $80 \%$ de las mujeres emplea los morfemas genéricos, respectivamente. En el segundo caso, tanto hombres y mujeres bilingües representan $90 \%$ del total de uso.

En la tercera generación, hombres y mujeres únicamente utilizan la forma quechua, que está representada por el morfema libre correspondiente, es decir, no tiene marca y se le denomina morfema $\theta$.

En los niveles de instrucción tanto en primaria, secundaria y superior, los porcentajes de uso del morfema de género español son también notables, tal como ocurre en la primera y segunda generación (90 y 80\%, respectivamente). En la tercera generación, casi $100 \%$ de hablantes utilizan las formas quechuas, excepto en el nivel secundario donde aparece $10 \%$ de incidencia para el morfema genérico del español.

\section{b) Interferencia por eliminación}

Ocurre cuando un rasgo gramatical existente en la lengua objetivo, antes del contacto con la lengua fuente, elimina una de sus formas: $-y k u /-n c h i s$.

Como ya señalamos anteriormente, en el quechua patrimonial el pronombre de primera persona plural tiene dos formas: nuqanchis (incluyente) que incluye al hablante y al oyente, y nuqayku (excluyente) excluye al hablante y a algunos oyentes. Las muestras que constituyen el producto de la investigación empírica nos indican que, en el valle del Colca, se viene generalizando el uso de la forma excluyente nuqayku. Este fenómeno de interferencia gramatical cuantitativa por eliminación de un morfema existente en la lengua quechua, antes del contacto con el español, ha sido eliminado por un proceso de neutralización.

El quechua distingue la oposición inclusiva (-nchis) y exclusivo (-yku) para el plural de primera persona: 
Inclusivo: nuqanchis takinchis allinta 'todos nosotros cantamos bien' Exclusivo: nuqayku takiyku allinta 'solo nosotros cantamos bien'

Actualmente existe la tendencia en el quechua de los bilingües del valle del Colca a usar $-y k u$ como plural de primera persona, eliminando la oposición inclusivo/exclusivo.

En el español, el morfema del pronombre de primera persona plural es nosotros; esto ha hecho que los hablantes bilingües del valle del Colca eliminen en su comunicación diaria la forma inclusiva -nchis. De esta manera, nuqayku albergará también el significado de nuqanchis:

(5) a) nuqayku tusuyku wititita

b) nuqayku puriyku chakraman

c) nuqayku sufriyku pasaqta

d) nuqayku takiyku allinta

e) nuqayku tapuyku

f) nuqayku tapusqayku

g) nuqayku Limata risaqku

h) nuqayku papata tarpuyku

i) nuqayku Kawana kuntita risaqku

j) nuqayku kusisqa kayku 'nosotros bailamos el wititi'

'nosotros vamos a la chacra'

'nosotros sufrimos mucho'

'nosotros cantamos bien'

'nosotros preguntamos'

'nosotros habíamos preguntado'

'nosotros iremos a Lima'

'nosotros sembramos papas'

'nosotros iremos a Cabanaconde'

'nosotros estamos felices'

La forma exclusiva $-y k u$ es usada con mayor intensidad por los hablantes bilingües del valle del Colca de la primera y segunda generación. Los hablantes mayores (tercera generación) mantienen aún en su comunicación cotidiana la forma inclusiva. Sin embargo, tal como indicamos en el análisis cuantitativo, muchos varones y mujeres de esta última generación utilizan la forma exclusiva. Es importante indicar que los hablantes bilingües del valle del Colca no diferencian el significado de los pronombres personales en el plural. Aquí se nota con mayor claridad la influencia permanente del español en este proceso de eliminación de la forma incluyente -nchis. 
Esta oposición incluyente y excluyente, como dijimos, es inexistente morfológicamente en el español; ello ha hecho que en el quechua arequipeño se extienda mayormente el uso de la forma excluyente que amalgama también el significado del morfema sufijal -nchis. De tal forma que el morfema - yku (yo, mi grupo, él y los oyentes) pasa a significar el sentido exclusivo e inclusivo:

(6) a) puriyku qhipanta

b) llapayku puriyku misaman

c) tarpuyku papata

d) tarpuyku arwihatapis

e) kayman hamuyku pusaqhurasta

f) munakuyku yachaywasiykuta 'andamos detrás de él'

'todos vamos a la misa'

'sembramos papas'

'también sembramos arvejas'

'aquí vamos a las 8:00 horas'

'amamos nuestro colegio'

Cuadro 7. Incidencia de interferencia gramatical cuantitativa por eliminación

$-y k u /-n c h i s$

\begin{tabular}{|c|c|c|c|c|}
\cline { 2 - 5 } \multicolumn{1}{c|}{} & \multicolumn{4}{c|}{$\begin{array}{c}\text { Generación 1 } \\
\text { (de 19 a 34 años) }\end{array}$} \\
\cline { 2 - 5 } \multicolumn{1}{c|}{} & \multicolumn{2}{c|}{ Hombre } & Mujer \\
\cline { 2 - 5 } \multicolumn{1}{c|}{} & $-\mathrm{yku}$ & -nchis & -nku & -nchis \\
\hline Primaria & $90 \%(18)$ & $10 \%(2)$ & $85 \%(17)$ & $15 \%(3)$ \\
\hline Secundaria & $80 \%(16)$ & $20 \%(4)$ & $75 \%(15)$ & $25 \%(5)$ \\
\hline Superior & $85 \%(17)$ & $15 \%(3)$ & $80 \%(16)$ & $20 \%(4)$ \\
\hline
\end{tabular}

Fuente: Elaboración propia.

El morfema plural de los pronombres de primera persona en español es 'nosotros' para el masculino y 'nosotras' para el femenino. En el quechua existen dos formas: inclusiva y exclusiva. Sin embargo, el pronombre plural exclusivo $-y k u$ se usa con mayor frecuencia e involucra al inclusivo -nchis; es decir, el exclusivo se refiere a todos de una manera general, al que habla y a los que participan en el proceso de la comunicación. 
En el cuadro 7 observamos que $90 \%$ de hombres y $85 \%$ mujeres de la primera generación con estudios primarios utiliza $-y k u$, y $85 \%$ de hablantes varones de la misma generación de nivel secundario y superior también prefieren en su interrelación el pronombre exclusivo. Las mujeres de nivel secundario y superior usan la forma exclusiva ( $75 \%$ y $80 \%$, respectivamente). Debemos indicar que en esta generación, tanto en primaria, secundaria como en superior, las mujeres bilingües prefieren la forma inclusiva -nchis, en mayor proporción a los hombres.

Cuadro 8. Incidencia de interferencia gramatical cuantitativa por eliminación $-y k u /-n c h i s$

\begin{tabular}{|l|l|l|l|l|}
\cline { 2 - 5 } \multicolumn{1}{c|}{} & \multicolumn{4}{c|}{$\begin{array}{c}\text { Generación 2 } \\
\text { (de 35 a 54 años) }\end{array}$} \\
\cline { 2 - 5 } & \multicolumn{2}{c|}{ Hombre } & \multicolumn{2}{c|}{ Mujer } \\
\cline { 2 - 5 } & -yku & -nchis & -yku & -nchis \\
\hline \multirow{2}{*}{ Primaria } & $75 \%(15)$ & $25 \%(5)$ & $70 \%(14)$ & $30 \%(6)$ \\
\hline Secundaria & $80 \%(16)$ & $20 \%(4)$ & $70 \%(14)$ & $30 \%(6)$ \\
\hline Superior & $80 \%(16)$ & $20 \%(4)$ & $75 \%(15)$ & $25 \%(5)$ \\
\hline
\end{tabular}

Fuente: Elaboración propia.

La forma exclusiva $-y k u$ se usa con mayor frecuencia en hablantes bilingües del valle del Colca de la segunda generación. Los hablantes varones con estudios primarios prefieren en el uso de $-y k u$ en $75 \%$; en secundaria y superior usan el morfema inclusivo $80 \%$ de hablantes.

Por otro lado, $80 \%$ de las mujeres de nivel primario optan por el uso del morfema $-y k u$, y en el nivel secundario y superior alcanzan el $70 \%$ y $75 \%$, respectivamente.

Estas imágenes diferenciadoras en el habla de las mujeres y los hombres nos permite indicar que la investigación sociolingüística ha demostrado que, en la variación lingüística, incide el factor sexo.

En suma, en todos los niveles de instrucción el porcentaje de uso de la forma exclusiva es elevado. 
Cuadro 9. Incidencia de interferencia gramatical cuantitativa por eliminación

$-y k u /-n c h i s$

\begin{tabular}{|c|c|c|c|c|}
\cline { 2 - 5 } \multicolumn{1}{c|}{} & \multicolumn{4}{c|}{$\begin{array}{c}\text { Generación 3 } \\
\text { (de 55 años a más) }\end{array}$} \\
\cline { 2 - 5 } \multicolumn{1}{c|}{} & \multicolumn{2}{c|}{ Hombre } & Mujer \\
\cline { 2 - 5 } & -yku & -nchis & -yku & -nchis \\
\hline Primaria & $50 \%(10)$ & $50 \%(10)$ & $50 \%(10)$ & $50 \%(10)$ \\
\hline Secundaria & $55 \%(11)$ & $45 \%(9)$ & $50 \%(10)$ & $50 \%(10)$ \\
\hline Superior & $60 \%(12)$ & $40 \%(8)$ & $60 \%(12)$ & $40 \%(8)$ \\
\hline
\end{tabular}

Fuente: Elaboración propia.

Los hablantes de la tercera generación mantienen las formas inclusiva y exclusiva. Aquí se observa que los morfemas plurales de primera persona preservan la estructura patrimonial del quechua.

Finalmente, en el español 'nosotros' sirve no solo para atestiguar la inclusión del interlocutor en una acción, sino para significar asimismo la implicación tanto del sujeto como de todos los participantes en ella. Como dijimos muchos hablantes bilingües del valle del Colca no diferencian las funciones de inclusión y exclusión de los pronombres de primera persona plural del quechua patrimonial, por lo que actualmente están optando por el uso de $-y k u$ como una estructura implicativa de valor enfático de la comunicación. Este recurso, en el que consideramos que están implícitas cuestiones de énfasis, no es ajeno al español, en el que el empleo del plural del pronombre de primera persona, tiene además otras funciones afines, como el plural mayestático, el plural de modestia o el plural asociativo, que indudablemente no tienen esa dimensión o significado en el quechua del valle del Colca.

\subsection{Interferencia gramatical cualitativa}

Este proceso de interferencia gramatical actúa en la lengua fuente de dos formas: por sustitución de sus elementos patrimoniales y por reestructuración tipológica. a) Interferencia morfológica por sustitución (-s / -kuna) 
Ocurre cuando los elementos estructurales de la lengua objetivo son reemplazados por formas de funcionalidad idéntica procedentes de la lengua fuente.

El morfema numérico quechua -kuna viene siendo sustituido por el morfema flexivo de número $-s$ de la lengua española. La forma ligada española se adiciona a los morfemas lexemáticos quechuas para formar nuevas palabras diferentes a las estructuras morfológicas del español y del quechua.

Quizá sea evidente que el hablante bilingüe del valle del Colca note la pérdida de prestigio del quechua y para exaltar su identidad adopte los morfemas de la lengua fuente. Estos usos no solo se están dando en esta región sino también en Argentina, Bolivia y otros países.

(7) warmis, 'mujeres'; allqus, 'perros' (Granda, 2001, p. 306).

Se observa que el morfema de número $-s$ español se cambia por el morfema plural quechua -kuna (warmis y allqus en reemplazo de warmikuna y allqukuna).

Aquí presentamos las interferencias gramaticales cualitativas por sustitución:

Morfema $-s$ en reemplazo de $-k u n a$

\begin{tabular}{|c|c|c|c|}
\hline akatanqa & 'escarabajo' & akatanqa $-\mathrm{s}$ & 'escarabajos' \\
\hline alqamari & ‘águila' & alqamari-s & ‘águilas’ \\
\hline apu & 'cerro' & apu-s & 'cerros' \\
\hline chukcha & 'cabello' & chukcha-s & 'cabellos' \\
\hline chuwa & 'plato' & chuwa-s & 'platos' \\
\hline sach'a & 'árbol' & sach'a-s & ‘árboles’ \\
\hline michi & 'gato' & michi-s & 'gatos' \\
\hline mayu & 'río' & mayu-s & 'ríos' \\
\hline warmi & 'mujer' & warmi-s & 'mujeres' \\
\hline kulihiyu & 'colegio' & kulihiyu-s & 'colegios' \\
\hline taruka & 'venado' & taruka-s & 'venados' \\
\hline
\end{tabular}




$\begin{array}{llll}\text { huk'ucha } & \text { 'ratón' } & \text { huk'ucha-s } & \text { 'ratones' } \\ \text { manka } & \text { 'olla' } & \text { manka-s } & \text { 'ollas' } \\ \text { p’asña } & \text { 'adolescente' } & \text { p'asña-s } & \text { 'adolescentes' }\end{array}$

Los casos de interferencia gramatical de tipo cualitativo que actúan sobre la lengua objetivo, determinando en ella procesos de sustitución de algunos de sus elementos estructurales por otros, de funcionalidad igual o similar, proceden también de la lengua fuente, que en este caso es el español:

$\begin{array}{lll}\text { (9) wasis } & \text { wasikuna } & \text { 'casas' } \\ \text { maquis } & \text { maquikuna } & \text { 'manos' } \\ \text { chaquis } & \text { chaquikuna } & \text { 'pies' } \\ \text { runas } & \text { runakuna } & \text { 'hombres' } \\ \text { allqus } & \text { allqukuna } & \text { 'perros' } \\ \text { wallpas } & \text { wallpakuna } & \text { 'gallinas' }\end{array}$

Incluso aparece el morfema flexivo en la estructura de otras categorías gramaticales (adverbios y conjunciones): manachus. Estos mismos fenómenos también ocurren en el quechua boliviano:

(10)porquechus, sichus (Sichra, 2003, p. 116).

También tenemos en el quechua del valle del Colca la presencia del morfema numérico español $-s$ al interior de oraciones nucleares:

(11) a) hamunqa tutas

b) paqariqtin wañun kawallirus

c) uywas waykurusqa

d) ñawpaq awilitus qarkan sumaqpuni 'antes los abuelitos eran buenos'

e) askha chakras chay wichaypi 'varias chacras hay en la parte alta'

f) runas mihunku 'vendrán en las noches'

'al amanecer murieron los caballeros'

'los animales habían entrado'

'los hombres comían'

En a, b, c, d, e y f el hablante bilingüe usa el morfema numérico español $-s$, en reemplazo del plural quechua -kuna. Las formas de interferencia gramatical 
cualitativa por sustitución son de uso generalizado entre los hablantes de la comunidad bilingüe del valle del Colca.

Esta influencia del español en el quechua no depende exclusivamente del prestigio de la lengua dominante, sino también de una serie de factores sociolingüísticos, como la intensidad del contacto y la duración del mismo. Actualmente, el valle del Colca es uno de los lugares más visitados por turistas nacionales e internacionales, e, incluso, las vías de comunicación han mejorado y el encuentro entre hablantes de lengua española y quechua ha crecido por cuestiones de turismo y comercio en general.

Cuadro 10. Incidencia de interferencia gramatical cualitativa por sustitución -s / -kuna

\begin{tabular}{|c|c|c|c|c|}
\cline { 2 - 5 } \multicolumn{1}{c|}{} & \multicolumn{4}{|c|}{$\begin{array}{c}\text { Generación 1 } \\
\text { (de 19 a 34 años) }\end{array}$} \\
\cline { 2 - 5 } & \multicolumn{2}{c|}{ Hombre } & Mujer \\
\cline { 2 - 5 } & $-\mathrm{s}$ & - kuna & $-\mathrm{s}$ & -kuna \\
\hline Primaria & $25 \%(5)$ & $75 \%(15)$ & $25 \%(5)$ & $75 \%(15)$ \\
\hline Secundaria & $25 \%(5)$ & $75 \%(15)$ & $20 \%(4)$ & $80 \%(16)$ \\
\hline Superior & $30 \%(6)$ & $70 \%(14)$ & $30 \%(6)$ & $70 \%(14)$ \\
\hline
\end{tabular}

Fuente: Elaboración propia.

El morfema numérico -kuna del quechua del valle del Colca es sustituido por la forma española $-s$ en diversos niveles. En la primera generación (hablantes varones y mujeres con nivel primario), la incidencia del morfema plural español alcanza 25\%; proporción elevada de sustitución. En el nivel secundario y superior tenemos $25 \%$ y $30 \%$ de uso en el caso de los varones y $20 \%$ y $30 \%$ en las mujeres. Esto significa que hombres y mujeres de esta generación mantienen aún el morfema -kuna en su comunicación cotidiana, pero la influencia del español en el quechua del valle del Colca crece no solo por el contacto de lenguas, sino por el desarrollo del turismo en Caylloma y el nivel de instrucción de los hablantes. El uso del morfema numérico español $-s$ se viene generalizando en estudiantes bilingües de institutos superiores de esta región, y también en alumnos bilingües de nivel universitario. 
Cuadro 11. Incidencia de interferencia gramatical cualitativa por sustitución -s/-kuna

\begin{tabular}{|c|c|c|c|c|}
\hline & \multicolumn{4}{|c|}{$\begin{array}{c}\text { Generación } 2 \\
\text { (de } 35 \text { a } 54 \text { años) }\end{array}$} \\
\hline & \multicolumn{2}{|c|}{ Hombre } & \multicolumn{2}{|c|}{ Mujer } \\
\hline & $-s$ & -kuna & $-s$ & -kuna \\
\hline Primaria & $20 \%(4)$ & $80 \%(16)$ & $15 \%(3)$ & $85 \%$ (17) \\
\hline Secundaria & $20 \%(4)$ & $80 \%(16)$ & $10 \%(2)$ & $90 \%$ (18) \\
\hline Superior & $20 \%(4)$ & $80 \%(16)$ & $15 \%(3)$ & $85 \%(17)$ \\
\hline
\end{tabular}

Fuente: Elaboración propia.

En la generación 2 (hablantes varones y mujeres de 35 a 54 años), la presencia del morfema de número $-s$ disminuye un tanto, ya que su aparición alcanza en el nivel primario $20 \%$ en hombres y $15 \%$ en mujeres. En el nivel secundario, $20 \%$ y $10 \%$ en hombres y mujeres, respectivamente. $Y$ en el nivel superior, 20 y $15 \%$. Aquí se observa que tanto en hombres como en mujeres la incidencia del morfema quechua patrimonial se mantiene en la comunicación. Son las mujeres quienes, en diversos dominios sociolingüísticos, prefieren en mayor proporción la forma quechua -kuna.

Cuadro 12. Incidencia de interferencia gramatical cualitativa por sustitución -s/-kuna

\begin{tabular}{|c|c|c|c|c|}
\hline & \multicolumn{4}{|c|}{$\begin{array}{l}\text { Generación } 3 \\
\text { (de } 55 \text { a más) }\end{array}$} \\
\hline & \multicolumn{2}{|c|}{ Hombre } & \multicolumn{2}{|c|}{ Mujer } \\
\hline & $-s$ & -kuna & $-S$ & -kuna \\
\hline Primaria & $5 \%(1)$ & $95 \%(19)$ & $0 \%(0)$ & $100 \%(20)$ \\
\hline Secundaria & $10 \%(2)$ & $90 \%(18)$ & $5 \%(1)$ & $95 \%$ (19) \\
\hline Superior & $10 \%(2)$ & $90 \%$ (18) & $0 \%(0)$ & $100 \%(20)$ \\
\hline
\end{tabular}

Fuente: Elaboración propia. 
En la tercera generación, el uso del morfema numérico español disminuye completamente en todos los niveles.

En suma, la sustitución del morfema numérico quechua -kuna por la forma $-s$ de la lengua española es importante en el quechua del valle del Colca por el contacto intenso de las lenguas materia del presente trabajo de investigación.

Por último, las diferencias de uso del morfema numérico $-s$ en el quechua de hablantes bilingües del valle del Colca son particularmente visibles; las generaciones mayores prefieren el morfema patrimonial quechua -kuna; y los jóvenes muestran una inclinación hacia la forma española. Los hablantes de esta comunidad de habla son más conservadores en el uso de morfemas y palabras quechuas, conforme avanza la edad correspondiente.

\section{Conclusiones}

1. En Arequipa, y específicamente en el valle del Colca, el contacto entre el español y el quechua ha creado fenómenos no solo de bilingüismo individual, sino también de bilingüismo social. Los hablantes de esta comunidad de habla - o al menos parte de ellos - mantienen las dos lenguas en su repertorio verbal; estas lenguas, en el país, se hallan desequilibradas funcionalmente, de manera que en ciertos dominios sociales se propicia el uso del español en detrimento del quechua.

2. El bilingüismo permanente e intenso entre el español y el quechua del valle del Colca ha sido caldo de cultivo para la presencia de una serie de interferencias o transferencias lingüísticas de la lengua fuente a la lengua objetivo. Estas marcas transcódigas entendidas como cualquier rasgo lingüístico que se introduce en una lengua A por la influencia de una lengua B, configuran una variedad singular en el quechua de los hablantes bilingües.

3. Los fenómenos de interferencia gramatical pueden ser de índole cuantitativa, que conllevan a la modificación del inventario de rasgos gramaticales existentes en la lengua objetivo (bien por adopción de uno nuevo, procedente de la lengua fuente, bien por eliminación de uno propio) y los de carácter cualitativo, que no varían cuantitativamente dicho inventario, pero sí la estructura de la lengua. 
4. Los casos de interferencia gramatical cuantitativa (por adopción -o, $-a /(-u$, $-a /$ y eliminación -nchis / -yku) y cualitativa (por sustitución $-s /-k u n a$ ), son formas que incrementan el vocabulario del quechua del valle del Colca y enriquecen la competencia lingüística y comunicativa del hablante bilingüe.

5. La especial situación de contacto en el valle del Colca ha determinado el cambio lingüístico en dirección del español. Es decir, la comunidad quechuahablante del Colca está llevando a cabo cambios, tomando como lengua meta el español. Este proceso, consecuencia del contacto, está determinando que el quechua vaya efectuando cambios en la fonología, morfología y la sintaxis. La morfología quechua del Colca actualmente muestra la adquisición de rasgos morfológicos en la flexión y la derivación que están orientados a la reestructuración gradual de la lengua nativa. En el proceso de influencia de la morfología flexional tenemos: adquisición de la oposición en el género masculino y femenino; el uso de un solo marcador del plural de primera persona $-y k u$ y sustitución de -kuna por -s. Estos procesos de interferencia están conduciendo a la reestructuración gramatical del quechua del valle del Colca.

6. El grado de interferencia gramatical cuantitativa y cualitativa de los hablantes bilingües del valle de Colca está determinado por las variables demosociales: edad, sexo y nivel de instrucción. Estas variables sociolingüísticas son factores extralingüísticos preeminentes de la interferencia gramatical. En todos los niveles se ha comprobado que las personas con menor instrucción producen un uso más cercano al quechua patrimonial, aumentando progresivamente la presencia de elementos españoles a medida que se incrementa el nivel de estudios. En lo referente a la influencia de la generación en los fenómenos de interferencia gramatical, la frecuencia de uso es inversamente proporcional a la edad de los informantes: los jóvenes son los que más usan las formas nuevas, y los mayores lo hacen en menor proporción. Finalmente, en los análisis que hemos realizado referentes a la división según el sexo de los informantes percibimos una mayor disposición de los varones a recibir las influencias de la lengua española, y cierta inclinación de las mujeres a los usos que más se acercan al quechua patrimonial. 


\section{Notas}

1 Las ediciones príncipes son de 1560 y 1608 , respectivamente.

2 Estos diccionarios y gramáticas quechuas se publicaron en 12 volúmenes dirigidos por Gary Parker en la serie Lengua y Sociedad del Instituto de Estudios Peruanos. Cubrieron el quechua Ancash-Huailas (Gary Parker y Amancio Chávez), el quechua Ayacucho Chanca (Clodoaldo Soto), el quechua Cajamarca-Cañaris (Félix Quesada), el quechua Cusco-Collao (Antonio Cusihuamán), el quechua Junín-Huanca (Rodolfo Cerrón-Palomino) y el quechua San Martín (David Coombs, Heidi Coombs, Robert Weber, Marinell Park, Nancy Weber y Víctor Cenepo).

3 "PRESEEA es un proyecto para la creación de un corpus de lengua española hablada representativo del mundo hispánico en su variedad geográfica y social. Esos materiales se reúnen atendiendo a la diversidad sociolingüística de las comunidades de habla hispanohablantes" (véase http://preseea.linguas.net/).

4 Ulloa Mogollón fue corregidor en la provincia de los collaguas en 1584. Dejó valiosa documentación sobre la historia del valle del Colca que fue publicada por Marcos Jiménez de la Espada en sus Relaciones geográficas de Indias, en 1881.

\section{Referencias bibliográficas}

Adelaar, W. (1977). Tarma Quechua: Grammar, Texts, Dictionary. Lisse: Peter de Ridder Press.

Albó, X. (1974). Los mil rostros del quechua. Sociolingüistica de Cochabamba. Lima: Instituto de Estudios Peruanos.

Callata, N. \& Cervantes, M. (1999). Relación entre el contacto de lenguas ( $Q$ C) y la formación del léxico híbrido en el quechua de los pobladores de Pinchollo, anexo del distrito de Cabanaconde, Caylloma (Tesis de licenciatura). Universidad Nacional de San Agustín de Arequipa, Perú.

Callo Cuno, D. (2015). Interferencia gramatical de hablantes bilingües en el valle del Colca (Tesis para optar el grado de Doctor en Lingüística). Universidad Nacional Mayor de San Marcos. Facultad de Letras y Ciencias Humanas, Lima, Perú.

Cerrón Palomino, R. (1987). Lingüistica quechua. Cusco: Centro de Estudios Rurales Andinos Bartolomé de Las Casas. 
Cusihuamán, A. (1976). Gramática Quechua: Cuzco-Collao. Lima: Editorial Ministerio de Educación.

De Santo Tomás, F. Domingo. (1996 [1560]). Grammatica o arte de la lengua general de los indios de los reynos del Perú. Estudio introductorio y notas por Rodolfo Cerrón Palomino. Cusco: Centro de Estudios Regionales Andinos Bartolomé de Las Casas.

Escobar, A. M. (2000). Contacto social y lingüístico. El español en contacto con el quechua en el Perú. Lima: Pontificia Universidad Católica del Perú.

Fishman, J. (1965). Who Speaks What Language to Whom and When? La Linguistique, 1, 2, 67-88.

González Holguín, D. (1993 [1608]). Vocabulario de la lengua quichua, 2 tomos. Quito: PEBI, Corporación Editora Nacional.

Granda, G. (2001). Estudios de lingüistica andina. Lima: Pontificia Universidad Católica del Perú.

Gugenberger, E. (1995). Conflicto lingüístico: el caso de los quechua hablantes en el sur del Perú. En K. Zimmermann (Ed.), Lenguas en contacto en Hispanoamérica. Nuevos enfoques (pp. 183-202). Madrid: Iberoamericana.

Manrique, N. (1985). Colonialismo y pobreza campesina. Caylloma y el Valle del Colca siglos XVI-XX. Lima: Centro de Estudios y Promoción de Desarrollo-DESCO.

Payrató, Ll. (1985). La interferencia lingüística. Barcelona. Editorial CurialPublicacions.

Parker, G. (1963). La clasificación genética de los dialectos quechuas. Revista del Museo Nacional, XXXII, 241-252.

Pease, F. (1977). Collaguas I. Lima: Pontificia Universidad Católica del Perú.

Quesada, F. (2007). Formación del léxico quechua. En Actas del II Congreso Internacional de Lexicología y Lexicografía Pedro Bembenutto Murrieta. Lima: Academia Peruana de la Lengua. 
Sichra, I. (2003). La vitalidad del quechua. Lengua y sociedad en dos provincias de Cochabamba. La Paz: PROEIB-Andes, Plural Editores.

Siguan, M. (2001). Bilingüismo y lenguas en contacto. Madrid: Alianza Editorial.

Soto Ruiz, C. (2006). Quechua, manual de enseñanza. Lima: Instituto de Estudios Peruanos.

Taylor, G. (1984). Yauyos: Un microcosmos dialectal quechua. Revista Andina, 2, 1, 121-146.

Thomason, S. y Kauffman, T. (1988). Language Contact, Creolization and Genetic Linguistics. Berkeley: University of California Press.

Torero, A. (1964). Los dialectos quechuas. Análisis Cientificos de la Universidad Agraria II, 4, 446-478.

Torero, A. (1972). Lingüística e historia de los andes del Perú y Bolivia. En A. Escobar (Comp.), El reto del multilingüismo en el Perú. Lima: Instituto de Estudios Peruanos.

Torero, A. (1974). El quechua y la historia social andina. Lima: Universidad Ricardo Palma.

Ulloa de Mogollón, J. (1965) [1586]. Relación de la provincia de los Collaguas, para la descripción de las Indias que su magestad manda hacer. En Relaciones geográficas de Indias, vol. I, editado por Marcos Jiménez de la Espada (pp. 326-333). Madrid: Biblioteca de Autores españoles.

Weinreich, U. (1953). Languages in Contact. La Haya: Mounton [traducción al español: Lenguas en contacto. Descubrimientos y problemas. Caracas: Ediciones de la Biblioteca de la Universidad Central]. 